\title{
Resource guides for astronomy educators and their students
}

\author{
Andrew Fraknoil,* \\ ${ }^{1}$ Fromm Institute, University of San Francisco \\ *fraknoiandrew@fhda.edu
}

\begin{abstract}
This is a brief review of my long-term project to create annotated resource guides for astronomy educators and their students. The college-level guides focus on a range of astronomical topics, such as "Women in Astronomy," "Science Fiction and Astronomy," "Black Lives in Astronomy," "Music Inspired by Astronomy," "Light Pollution, Radio Interference, and Satellite Swarms," etc. Their aim is to bring together pointers to a range of reliable and interesting materials for instruction, discussion, or student projects in one convenient and easy-to-find place.
\end{abstract}

Keywords: Resources; Interdisciplinary; Bibliographies; Instructional Materials; Journals; Diversity

\section{Origin and History of the Project}

The resource guides began to take shape when the author was editor of Mercury magazine at the Astronomical Society of the Pacific (ASP) during the 1970's and 1980's. Rather than featuring individual book reviews, which other astronomical publications were already doing, we hit on the idea of reviewing introductory materials on a specific topic, and including magazine articles as well as books. This was before the World Wide Web existed, when finding relevant materials for teaching or outreach was more laborious.

Among these first efforts were:

- A Subject Index to Astronomy in Scientific American Magazine (Fraknoi, 1977b)

- Interdisciplinary Approaches to Astronomy (Fraknoi, 1977a)

- The Sun: A Reading List (Fraknoi, 1977c)

- Close Encounters with [Astronomical] Pseudoscience (Fraknoi, 1978)

- Computer Programs in Astronomy (Mosley and Fraknoi, 1985)

Eventually, the guides became independent of the magazine, and were printed (and, occasionally, specially written) for workshops for teachers (in the ASP's Universe in the Classroom series) and symposia for Astronomy 101 in structors (in the ASP's Cosmos in the Classroom series.) Later, some of those guides were collected in the ASP's The Universe at Your Fingertips, which became available as a DVD-ROM (https://myasp.astrosociety.org/product/DV122/ the-universe-at-your-fingertips-20-dvd-rom).

The contents of the guides began to cover a wider range of subjects, including a longer resource covering the contributions of women to astronomy and a catalog of music inspired by astronomy.

In the decade of that began in 2011 , some of the existing guides, and many new ones, were moved to the ASP's webpage (now at: https://astrosociety.org/education-outreach/ education-resources.html) and also to the web page: http:// www .fraknoi .com/resource-guides-on-astronomy-education/.

More recently, the most-often requested guides have been assigned convenient, short URLs, using the bit.ly notation. In the next section, we list and explain some of the guides that seem to be the most useful for educators. Bear in mind that all the guides focus on non-technical materials, so that they are accessible to both educators and students. And all the guides are limited to materials available in English. 


\section{Current Guides Available on the Web}

\subsection{Guides Related to Diversity}

The Contributions of Women to Astronomy: http://bit ly/astronomywomen gives resources on the general issues that women have faced (and continue to face) in astronomy, and then suggests books, articles, and webpage about the life and work of 37 women: 20 living scientists, and 17 noted women of the past. In addition, there are one or two resources for another 24 women about whom less non-technical material is available.

Black Lives in Astronomy: http://bit.ly/blackastro provides published and web resources on the lives and work of 26 black astronomers in North America, selected because material about them was easily available to students. It begins with a short section on the general topic of the challenges and triumphs of black astronomers.

The Astronomy of Many Cultures: http://bit.ly/ astrocultures is designed to make U.S. students more familiar with the astronomical contributions and thinking of cultures outside North America and Europe. While it is no way complete, it does provide materials for an introduction to the astronomy of the indigenous people of Central and South America, Asia, Africa, Polynesia, and Australia.

\subsection{Guides to Interdisciplinary Approaches}

Science Fiction Stories with Good Astronomy: http://bit.ly/ astroscifi is an annotated catalog of written science fiction that has reasonable science as its basis. It is organized by astronomy topic, so you can look up good novels and stories to recommend on black holes, Mars, or SETI, for example. A number of the short stories are now available on the Web and URLS are provided

Music Inspired by Astronomy: http://bit.ly/ astronomymusic is another annotated catalog, of pieces of music that are based on serious astronomy (and not just a casual mention of the Moon.) It includes both classical and popular music, and is organized by astronomy topic. It is limited to pieces that are available on disk or on the Web. Don't miss the Supernova Sonata, the Hubble Cantata, or the five rock and roll songs about black holes!

Plays and Films Inspired by Astronomy: http://bit.ly/ astroplays is a shorter listing of selected plays and movies (in English) that feature astronomers as characters or revolve around astronomical ideas or history. This list is in its first editions and needs additional suggestions.

Responding to Astronomical Pseudo-science: http:// bit.ly/astropseudoscience is a guide to resources to help astronomers or their students learn the skeptical, rational perspective about such "fiction sciences" as astrology, UFOs as alien spaceships, ancient astronauts, crop circles, creationism (or intelligent design), astronomical doomsdays, and much more.

(Note: In 2021, particularly in the U.S., an enormous amount of gullible media attention is being paid to military images and videos that are claimed to show mysterious spaceships with unexplainable behavior. But investigators have found earthbound explanations that are much more reasonable. A new one-page guide to web resources on these and other UFO issues is newly available at: http://bit.ly/ufoskeptic)

This Day in Astronomical History: http://bit.ly/ astrodates is a month-by-month calendar of 158 astronomical anniversaries or events to enrich a class or talk, including a number involving work by women. (And, unlike most such calendars, the emphasis is not on human spaceflight anniversaries, but on real astronomical research.)

\subsection{Guides for Teaching Astronomy}

Doing Astronomy Outreach and Education When You Can't Go Out: http://bit. ly/astrooutreach is a recent compilation of resources for doing remote education and outreach (both by necessity during the pandemic, and by choice at other times.) The guide also includes other lists of resources created by veteran educators and by crowdsourcing.

Short Astronomy Videos to Go with Each Chapter of OpenStax Astronomy: http://bit.ly/shortastronomyvideos is an annotated listing of free videos in English, available on the web, each shorter than 10 minutes, to help illustrate and illuminate astronomy concepts. The catalog is keyed to the free, on-line, open-source, introductory astronomy textbook for which I am the lead author, but can be used with any other course materials as well. Many NASA and ESA videos are included and organized by topic.

Sources of Astronomy Images: http://bit.ly/ astronomyimages is a brief, incomplete list of where beginners in astronomy education can find the largest catalogs of astronomy images to use in their work. I apologize that the emphasis is on sources where captions are available in English.

M.O.O.S.E: Menu of Outreach Opportunities for Science Education: http://bit.ly/AASMOOSE (all caps) is a web docu ment, put together for the American Astronomical Society's Astronomy Ambassadors program, to provide early-career astronomers with resources, guidelines, and opportunities for doing outreach. The focus is on the US, but the majority of resources could be adapted for astronomy outreach in other countries.

Astronomy and Humor: http://bit.ly/astrohumoris a short selective list of sources for astronomical jokes, cartoon, and other humorous materials - to spice up a talk, a final exam, or post-conference visit to a bar.

\subsection{Miscellaneous Guides}

Light Pollution and Dark Skies : http://bit.ly/darkskyguide is beginner's guide to issues where human activities are pos ing a problem for astronomical research. Designed, as all these guides are, so students can also use them, it includes nontechnical explanations of the problem and proposed solutions. Other topics included are radio interference and satellite swarms.

Pluto and the Kuiper Belt: http://bit.ly/plutokuiper is a 2019 guide to introductory readings about Pluto the dwarf planet, the controversy of its classification, and its exploration by New Horizons. It also focuses on the Kuiper Belt, the flyby of the KBO previously known as Ultima Thule (now officially called Arrokoth) and the first claims of a Planet Nine in the belt.

An Index to the ASP's Mercury Magazine: http://bit.ly/ mercuryindex is a listing of all the main articles that appeared from 1972 through 2019 in the Astronomical Society of the Pacific's popular-level magazine. You can find collections of Mercury in all the libraries that have the journal Publications of the ASP. Many noted astronomers explained their work for the nonscientist in the pages of this magazine.

Recently, I have put together a one-page catalog with links to all my active resource guides in one convenient place. You can find it at: http://bit.ly/fraknoiguides

\section{Other Guides and Future Efforts}

Between 2001 and 2013, I served as Founding Co-editor, and later as editorial consultant, for Astronomy Education Review, a refereed journal on astronomy education, published first by the National Optical Astronomy Observatories and then 
by the American Astronomical Society. A subject index to all the published issues is available at: https://aas.org/teach/ subject-index-papers-astronomy-education-review-2001-2013 It can serve as a good introduction to the state of astronomy education research in those years. A number of other guides, some now a few years old, are featured at the two web addresses given toward the end of section 1 , above. I try to update each of the guides every few years or so, but it's a bit random when each one has its turn. I very much welcome suggestions for any of these guides. My hope is that they can, in some small way, make the efforts of astronomy educators more effective and point them in useful directions.

\section{References}

Fraknoi, A. (1977a). Interdisciplinary approaches to astronomy. Mercury, 6(5):20.

Fraknoi, A. (1977b). Subject index to astronomy in scientific american (1960-1976). Mercury, 6(1):20.

Fraknoi, A. (1977c). The sun: A reading list. Mercury, 6(4):20.

Fraknoi, A. (1978). Close encounters with pseudoscience. Mercury, $7(1): 21$.

Mosley, J. and Fraknoi, A. (1985). Computer programs in astronomy. Mercury, 14(1):27. 